\title{
The influence of NKOR, sex, and task on visual pattern discrimination
}

College students learned two visual discriminations, one designed to be easier for males, and one designed to be neutral in difficulty for the sexes. One group learned in the typical "be correct" situation, with knowledge-of-results (KOR) on each trial. The other group learned to "be consistent" with no KOR (NKOR). KOR was not helpful, and results suggested that it may be detrimental when the task is difficult and consists primarily of perceptual differentiation.

Most discrimination experiments with human Ss, e.g., Terrell \& Kennedy, 1957, show facilitation from reinforcers in the form of fewer trials or errors. Occasional reports of detrimental reinforcement effects in learning have appeared in the literature during the last four decades (Ellis \& Pryer, 1958; Hillix \& Marx, 1960; Miller \& Estes, 1961). Miller and Estes showed that 9-year-old boys made fewer errors with knowledgeof-results (KOR) only than with KOR plus a monetary reward of 1 cent or 50 cents for each correct response. Results of a replication with adults were equivocal with respect to the reinforcement effect, but produced clear sex differences (females made fewer errors) and a sexincentive interaction (Estes, Miller, \& Curtin, 1962). The obtained sex differences were thought to be due to the nature of the task, which consisted of drawings of faces. This required verification. But the results of both studies suggested a more interesting question: If reinforcement can be detrimental to learning, can knowledge-of-results also be detrimental?

The term "knowledge-of-results" is usually understood to collect a class of post-response stimuli which provide the $S$ with information regarding the correctness of his response. In this sense, the notion of detrimental KOR is a logical contradiction. A large amount, or more precise information, ought to improve learning. In perceptual learning it may not. In a recent report on a study of recognition of disoriented figures (Braine, 1965) commented that " ... rapid learning occurred in the complete absence of knowledge of results. It is clear that the effects of repeated exposure are essentially the same in the groups with different kinds of information."

The experiment reported here was designed to: (1) establish a discrimination task neutral in difficulty for the sexes; (2) establish a task easier for males; and (3) to compare learning of a two-choice pattern discrimination when there is no knowledge-of-results (NKOR) with learning when KOR is given. Clearly, the $S$ who receives NKOR cannot be expected to choose a stimulus arbitrarily selected as "correct" by E. However, he can learn to choose the same stimulus consistently. In a sense these two tasks may not be precisely the same. It is reasonable to ask, however, whether Ss can learn to differentiate and identify two initially indistinguishable patterns more easily with NKOR or in the traditional discrimination paradigm with KOR.

\section{Method}

The task. The discrimination task designed to be easier for males consisted of line drawings of a car as seen from the rear. The only difference between the two was in the positioning of the tail-lights, which were slightly higher on one than the other. The task designed to be of equal difficulty for the sexes consisted of black-and-white checker-board designs-a grid of 64 small squares. The difference between the two was in the position of one small black square within the larger pattern. Two of these pairs of Checked Patterns were used, designated Pattern I and Pattern II. These were constructed to be equivalent in difficulty, and were found to be in preliminary testing, but this experiment was expected to verify their equivalence.

Subjects. Ss were introductory psychology students at the University of Louisville. There were $24 \mathrm{Ss}$ in the NKOR group and 32 in the KOR group.

Design and Procedure. All Ss learned both the Car and a Checked Pattern, with order of presentation balanced in both NKOR and KOR conditions. The design was thus a mixed factorial with four variables: KOR, Sex, Order, and Task. In the NKOR group Ss were instructed to "be consistent." Ss merely stated "left" or "right" and E made no comment. In the KOR group Ss were told that one of the two drawings was correct, and a red light would appear following each correct response. Responses were made by turning a switch to the right or left. The second task was performed by each $S$ immediately after the first. Criterion was 16 correct (KOR) or consistent (NKOR) cholces. The apparatus was a simple tachistoscope in which the designs were exposed for an interval of $1.2 \mathrm{sec}$. on each trial.

Results

The first analysis was made of errors on the Checked Patterns in the KOR group to determine whether Pat-

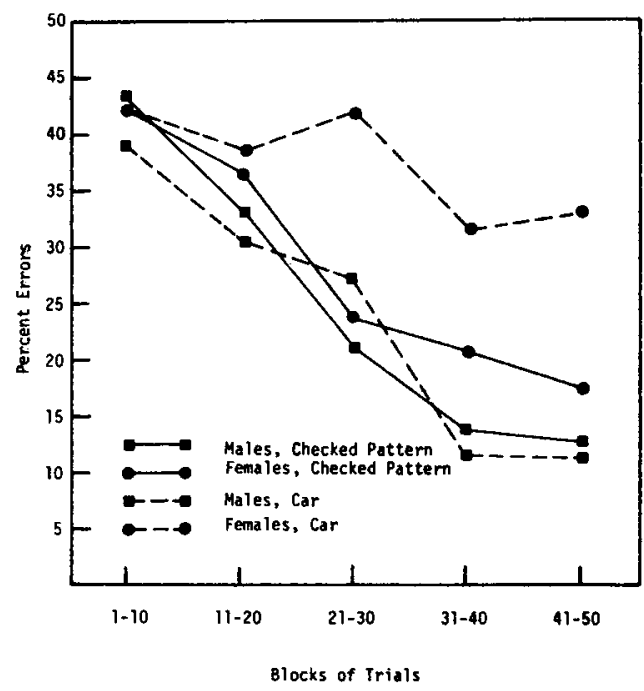

Fig. 1. Sex differences as a function of task-car vs. checked pattems. (KOR Group only) 
Table 1. Mean Errors KOR Condition

\begin{tabular}{lcc} 
Task & Moles & Females \\
\hline Car & 15.38 & 23.69 \\
Checked Pattern & 15.62 & 14.75 \\
\hline
\end{tabular}

terns I and II were actually equivalent. Pattern was not a significant source of variance, and subsequent analyses were made without regard to Pattern difference.

Overall analysis of errors in the KOR group showed Sex to be a significant source of variance $(F=4.06$, $\mathrm{df}=1 / 60, p=.05$ ). Females did very poorly on the Car (Fig. 1) even after practice with the Checked Patterns. Table 1 shows the error means for KOR Ss. The sexes did not differ on the Checked Patterns, and both did much better after practice with the Car. Separate analyses for males and females support these results. Apparently the Car discrimination was easier for males, and the Checked Patterns were of equivalent difficulty for the two sexes.

Comparison of learning with NKOR and KOR complicates the picture. KOR was certainly not helpful. The percentage of slow learners who had not completed criterion run at the end of 50 trials was higher in the KOR group $-55 \%$ as compared with $46 \%$ in the NKOR group; chi square for contingency in all eight cells was 80.44, $p=<.001$. This difference is greater, of course, if one considers only the first task, learned without practice $-68 \%$ required 50 trials for $\mathrm{KOR}, 45 \%$ in the NKOR condition. The mean number of trials for KOR Ss on the first task was 44.57 ; for NKOR Ss the mean was 42.35. Because there was a significant negative correlation between the means and variances $(r=.8446, \mathrm{df}=14$, $\mathrm{p}=<.01$ ) analysis of variance of trials was not used.

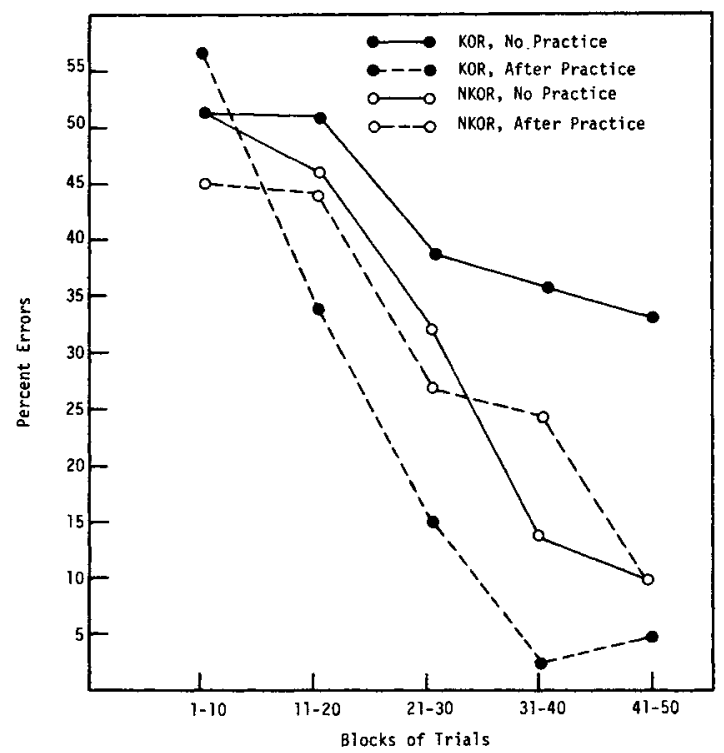

Fig. 2. Effect of NKOR and KOR on checked patterns. ("Errors" in NKOR group represent choices of pattern not selected for criterion run.)
(When many Ss required 50 trials, the mean was high, the variance low, and vice versa.) Inspection of subgroups, however, suggests that the Car was less difficult for females if they were given NKOR.

\section{Discussion}

If the possibility of detrimental KOR is accepted, how can this be explained? Restle (1955) has suggested that the typical discrimination task consists in part of a perceptual differentiation, and in part of a paired-associates task, in which the Slearns to associate a particular stimulus with reinforcement. The proportion of total trials on which these two tasks occupy the Sprobably varies with age and intelligence of Ss, number of choices available, and other variables. An animal, mentally-retarded individual, or even a normal child, might spend many trials learning to associate a stimulus with reinforcementeven when there are only two choices. A human adult, once he could differentiate between them, would surely not require more than oneor at the most, two-trials to learn which is the "correct" one. Our task for both NKOR and KOR Ss was clearly one consisting almost entirely of perceptual differentiation. During this portion of the task, KOR providing information as to the correctness of the overt choice might conceivably be detrimental.

Although it is generally ackowledged that learning curves based on errors, averaged over Ss, may be ambiguous, such curves do reflect the gradual elimination of learners, and group comparisons made in this way are often instructive. In order to construct such curves for NKOR Ss, an "error" score was devised in the following way: Whichever design $\mathrm{S}$ was choosing when he became consistent was considered the "correct" one for him, and data sheets were scored accordingly. It is at leastpossible that Ss in the NKOR group selected for criterion run the stimulus which they had previously reponded to most often. Ss who had not become consistent at the end of 50 trials were assigned a chance frequency of five errors per block of 10 trials. The "learning curves" obtained in this way are plotted together with learning curves based on "true errors" for KOR Ss in Fig. 2. It may be objected that these "error" scores are meaningless when Ss are free to choose either stimulus; however, the same objection can be made regarding Ss in the KOR condition prior to criterion-their responses may not reflect choices until they can distinguish between the two patterns. It is interesting, at least, that curves based on "true errors" and curves based on pseudo-errors have the same general appearance.

If these curves are taken seriously, it is apparent that the poorest group, the atypical group, is the one in which KOR was given on the first task. The curves also suggest an interesting possibility-that there was no transfer or practice effect when Ss learned to be consistent with NKOR. The practice effect is quite obvious for KOR Ss. This is apparent also in the trial means. A conclusive statement as to the detrimental effect of KOR will require studies using the differential method, so that each $S$ can learn the two tasks known to be of equivalent difficulty, one under NKOR and one under KOR conditions; a larger number of Ss is indicated also, in order to reduce the percentage of $\mathrm{Ss}$ requiring all 50 trials and allow analysis of variance.

\section{References}

Braine, Lila Ghent. Disorientation of forms: An exmination of Rock's theory. Psychon. Sci., 1965, 3, 541-542.

Ellis, Norman R., \& Pryer, Margaret $W$. Primary versus secondary reinforcement in simple discrimination learning of mental defectives. Psychol. Rep., 1958, 4, 67-70.

Estes, B. W., Miller, L. B., \& Curtin, M. E. Supplementary report: Monetary incentive and motivation in discrimination learningsex differences. J. exp. Psychol, 1962, 63, 320.

Hillix, W. A., \& Marx, M. H. Response strengthening by information and effect in human learning. J. exp. Psychol., 1960, 60, 97-102.

Miller, L. B., \& Estes, B. W. Monetary reward and motivation in discrimination learning. $J$. exp. Psychol., 1961, 61, 501-504.

Restle, Frank. A theory of discrimination learning. Psych. Rev., 1955, 62, 111-119.

Terrell, Glenn, Jr., \& Durkin, Kathryn. Social class and the nature of the incentive in discrimination learning. J. abnorm. soc. Psychol., 1959, 59, 270-272. 